\title{
The Feasibility of Optimal Surgical Result Prediction according to the Center of Rotation Shift after Multilevel Cervical Total Disc Replacement
}

\author{
Jung Hwan Lee ${ }^{1}$, Jun Ho Lee ${ }^{2}$ \\ ${ }^{1}$ Department of Rehabilitation, Namdarun Rehabilitation Clinic, Seoul, Korea \\ ${ }^{2}$ Department of Neurosurgery, Kyung Hee University Medical Center, Seoul, Korea
}

Study Design: Retrospective design/spine clinic and tertiary referral hospital.

Purpose: This study investigated the association between shifted location of center of rotation (COR) and subsequent surgical outcomes and identified radiological parameters that corresponded to COR change after multilevel cervical total disc replacement (MCTDR).

Overview of Literature: Cervical total disc replacement (TDR) maintains normal cervical kinematics after surgery. However, there is a paucity of literature analyzing the relationship between radiological shifts of COR and subsequent clinical success, especially when it comes to the issue of MCTDR switch.

Methods: This study included 24 consecutive patients treated with MCTDR following the diagnosis of multilevel cervical disc herniation or stenosis. Numeric rating scale, range of motion (ROM) at both C2-7 segment and TDR implanted levels, and location of COR at TDR level were evaluated pre- and post-MCTDR. These parameters were compared between patients who experienced successful and unsuccessful pain relief.

Results: The inherent CORs relatively at ventrocranial coordinates demonstrated significant migrations to dorsocaudal location, more prominent shifts for the successful group, after MCTDR switch. The unsuccessful group showed markedly reduced C2-7 ROM and reduced angular improvement at C2-7 segment and MCTDR level compared with the successful group. Postoperative C2-7 ROM was related to postoperative COR along the $x$-axis.

Conclusions: Aside from ROM preservation at both C2-7 and TDR levels, COR restoration from ventrocranial location close to normal coordinates by posterior and inferior shifts was marked as a clinical success after MCTDR. The COR position along the x-axis after MCTDR was an important factor to determine maintenance of C2-7 ROM.

Keywords: Intervertebral cervical disc degeneration; Total disc replacement; Range of motion; Articular rotation

\section{Introduction}

Numerous reports have demonstrated the superiority of cervical total disc replacement (TDR) over standard anterior cervical discectomy and fusion (ACDF) on the preservation of concomitant physiological range of mo-

Received Jun 3, 2019; Revised Jul 19, 2019; Accepted Sep 10, 2019

Corresponding author: Jun Ho Lee

Department of Neurosurgery, Kyung Hee University Medical Center, 23 Kyungheedae-ro, Dongdaemun-gu, Seoul 02447, Korea

Tel: +82-2-958-8405, Fax: +82-2-958-8385, E-mail: moo9924@khu.ac.kr 
tion (ROM) at the implanted level and deterring adjacent segment pathology development after a single-level switch [1-5]. With these promising results, TDR has been increasingly used to address multilevel cervical spinal problems across institutions.

Multilevel cervical spine surgery is more challenging due to greater stress distribution on surgical and adjacent levels. Multilevel ACDF produces large stress or strain at adjacent levels [6,7], and multilevel cervical TDR (MCT$\mathrm{DR}$ ) is concievably a viable alternative to overcome these multilevel ACDF limitations.

Because the main purpose of cervical TDR is to maintain normal inherent kinematics of cervical spine after surgery, many investigations have focused on the maintenance of the original ROM and center of rotation (COR) at the TDR switched level. Although sporadic references assess COR shift after cervical single-level TDR performances [8-10], there is a paucity of literature analyzing the relationship between this radiological shift of COR and subsequent clinical success, especially when it comes to the issue of multilevel cervical TDR switch. It is important to define the influence of COR shift on the clinical outcomes after MCTDR to further determine an ideal coordinate for COR for optimal outcome achievement.

This study investigated whether a specific location of COR at cervical motion segment was associated with a particular clinical outcome and which radiological parameter corresponded to this COR shift after MCTDR.

\section{Materials and Methods}

\section{Patient selection}

This study was approved by the Institutional Review Board of Wooridul Spine Hospital (WRDIRB no., 201704-005). Informed consent was not required for this retrospective study including 24 patients who underwent MCTDR for multilevel cervical disc herniation or stenosis treatment. The subjects for MCTDR had two or three cervical disc levels with a mild degree of spondylosis, pathological disc bulging with thecal sac compression on every corresponding level, and dominant arm radiculopathy rather than axial neck pain. In such complicated situations that were unclear to isolate pathological level actually causing arm radiculopathy even with electrodiagnostic studies in addition to radiological evaluations, whole multilevel decompressive surgery was sometimes performed as part of a whole decompression concept to obtain clinical improvements. The inclusion criteria were as follows: (1) patients who had multilevel TDR between C3 and C4 and $\mathrm{C} 6$ and $\mathrm{C} 7$ for the treatment of neck and radicular pain, (2) no instability on dynamic flexion-extension radiograph $(<3 \mathrm{~mm})$, and (3) no response to conservative treatments for $>3$ months. The exclusion criteria were as follows: (1) patients with any history of cervical spine surgery, (2) infection, (3) metabolic or neoplastic bone disease, (4) ankylosis, (5) congenital anomaly of the spine, and (6) any trauma history associated with kyphotic (or lordotic) change or the tilt of curvature [11]. Patients who were excluded due to these medical histories or radiological reasons were recommended to undergo stabilizing surgery such as ACDF.

The participants included 17 males and 7 females (mean age, $52.3 \pm 8.1$ years). The involved segments were C3-4 and $\mathrm{C} 4-5$ in one case; $\mathrm{C} 3-4, \mathrm{C} 4-5$, and $\mathrm{C} 5-6$ in two cases; C4-5 and C5-6 in four cases; C4-5, C5-6, and C6-7 in three cases; and C5-6, 6-7 in 14 cases. All operations were performed by the same surgeon between February 2012 and December 2015.

\section{Device insertion}

The operating surgeon chose to use the artificial disc based on the morphology of endplates. Patients with greater concavity of the endplate contour received the Prodisc-C Vivo (DePuy Synthes, Raynham, MA, USA), whereas those with a relatively flat contour received the Baguera C (SpineArt, Geneva, Switzerland). After several trials before actual insertion of the real implants, $5-\mathrm{mm}$ tall devices with width and length that matched the size of endplates of each patient were chosen. The operating surgeon did not choose a device that would increase the new disc height by $>2 \mathrm{~mm}$ and confirmed the stable anchoring of the device after insertion. The midpoint between the bilateral longus colli muscles and the spinous process, visualized by intraoperative fluoroscopy, was used as the "midline" marker during the insertion of the device at each level. The superior and inferior plates of each device were inserted in-depth to reach as close to the posterior margin of the endplates at the corresponding level cervical vertebral body as possible under fluoroscopic guidance so that the core of the device is near the midpoint of the anterioposterior length of the intervertebral disc or endplates. 


\section{Clinical evaluation}

Pain was evaluated using the numeric rating scale (NRS), ranging from 0 (no pain) to 10 (worst possible pain). Each evaluation was assessed in the outpatient clinic before admission and during the final follow-up. All patients were asked to provide the average severity of their symptoms over the course of the previous week. The NRS record was assessed through an interview conducted by the assistant physician who was not involved during surgical treatments and was blinded to any radiographic outcomes. The first author who assessed these radiographic outcomes was not involved during surgical treatments and was blinded regarding NRS. The second author, the initial surgeon, was blinded regarding NRS and radiographic measurements. All 24 subjects were also blinded regarding NRS and radiographic outcomes.

Patients were divided into two groups: the successful and unsuccessful pain relief groups. Successful pain relief was characterized by at least a $50 \%$ reduction in NRS score in comparison with pretreatment [12].

\section{Radiologic evaluation}

C2-7 flexion and extension angles and TDR flexion and extension angles were defined as Cobb's angle measured from true lateral dynamic radiographs during full flexion and extension posture. The total $\mathrm{C} 2-7$ ROM and TDR ROM were obtained by summing up $\mathrm{C} 2-7$ or TDR flexion and extension angles.

A computer-assisted method was adopted to determine the location of COR at instrumented level. Images at full flexion and extension position were imported into GNU Image Manipulation Program ver. 2.8 (https://www.gimp. $\operatorname{org} /$ ) and were moved to superimpose their quadrilaterals of the lower vertebral body at the highest level among MCTDR level (e.g., C6 body for C5-6/C6-7 MCTDR). A coordinate system was established to show the determined location of the COR. The posterior superior corner of the lower vertebral body was set as the origin of coordinates, with the $\mathrm{x}$-axis directed forward along the superior endplate of the lower vertebral body and the $y$-axis directed vertically upward and perpendicular to the $\mathrm{x}$-axis; thus, the anterior direction on $\mathrm{x}$-axis and the cranial direction on $y$-axis were expressed as positive values [13]. The coordinates of points $\mathrm{A}, \mathrm{A}^{\prime}, \mathrm{B}$, and $\mathrm{B}^{\prime}$ were recorded at the superior endplate of upper vertebrae. $\mathrm{X}$ - and Y-coordinates

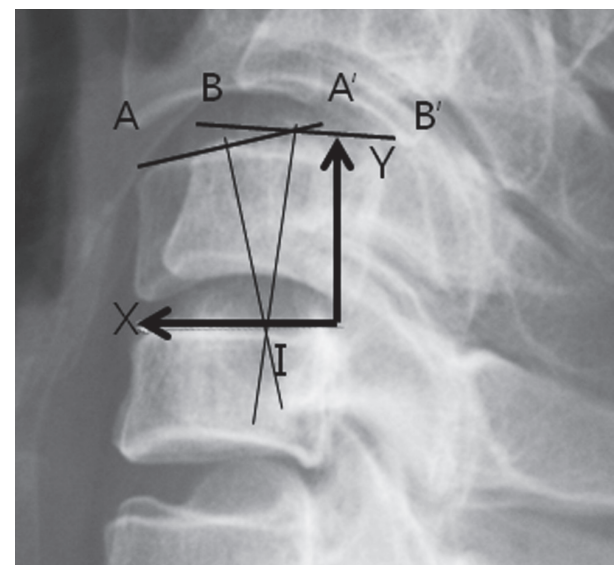

Fig. 1. Measurement of center of rotation on lateral radiographs by superimposing the flexion-extension simple radiography. $X$ and $Y$ indicate $X$ - and Y-coordinates, respectively.

of COR location (COR-X and COR-Y) were determined as intersection point, which was obtained by perpendicular lines drawn from midpoint of $\mathrm{AA}^{\prime}$ to $\mathrm{BB}^{\prime}$ (Fig. 1). The distance from the intersection point to origin of coordinates (posterior superior corner of the lower vertebral body) was calculated as percentages based on the width and height of the lower vertebral body (COR-X [\%], COR$\mathrm{Y}[\%]$ ) for offsetting individual variations in the sizes of the vertebrae. The size of the vertebra was evaluated based on the neutral position view. COR-X (\%) and -Y (\%) were obtained before and after MCTDR, and change in COR-X $(\%)$ and $-\mathrm{Y}(\%)$ were also calculated. COR-X (\%), COR-Y (\%), and change of COR-X (\%) and -Y (\%) were compared between the clinically successful and unsuccessful groups with respect to preoperation, postoperation, and angular change after MCTDR.

\section{Statistical analysis}

The SPSS ver. 14.0 statistical package (SPSS Inc., Chicago, IL, USA) was used for statistical analysis. All variables were tested using the Kolmogorov-Smirnov test to determine if the variables were normally distributed. Student $t$-test for normally distributed variables and MannWhitney $U$-test for not normally distributed variables were performed to determine differences in age, NRS, C2-7 angles, TDR angles, COR-X (\%), COR-Y (\%), and change in COR-X (\%) and -Y (\%) between the successful and unsuccessful surgical outcome groups. Moreover, binary logistic test was performed to evaluate the statistical significance of radiological variables in relation to the 
successful surgical outcomes. Linear regression test was conducted to assess statistical significance between change of COR and postoperative ROM of C2-7 and TDR level and postoperative COR and ROM of C2-7 and TDR level. Results were considered statistically significant if the $p$ values were $<0.05$.

\section{Results}

The mean follow-up period was $429.0 \pm 389.0$ days (range, 179-1,480 days). The mean preoperative NRS of $8.0 \pm 0.9$ significantly decreased to $3.9 \pm 1.2(p<0.001)$. Also, the mean pre-MCTDR COR-X and COR-Y of was $33.5 \% \pm 17.7 \%$ and $17.2 \% \pm 39.6 \%$, respectively, reduced significantly to $19.1 \% \pm 12.4 \%$ and $-16.8 \% \pm 79.1 \%$.

Sixteen patients $(66.7 \%)$ were designated to the successful outcome group and eight patients to the unsuccessful outcome group. Complications related to instrument failure, wound infection, neurologic damages, and severe blood loss following surgery or general anesthesia were not noted. Additionally, there was no difference in any
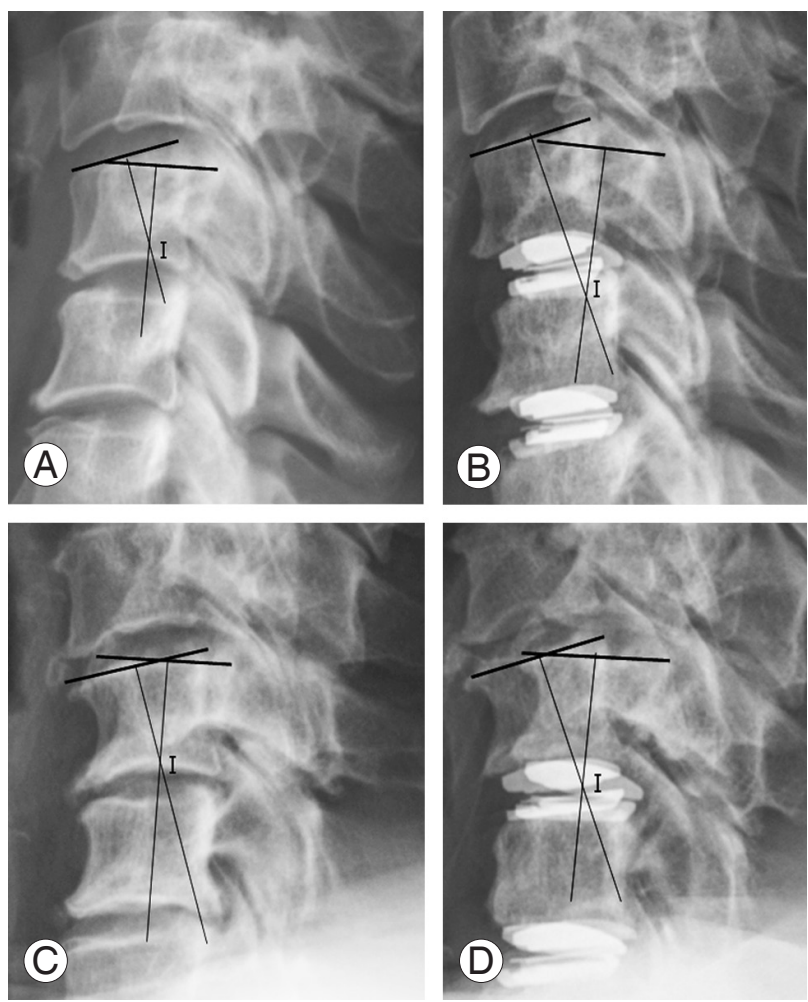

Fig. 2. Comparative demonstration of preoperative similar coordinates for center of rotation and its postoperatively differentiated shift between successful ( $A$, B) and unsuccessful subjects (C, D). radiological parameter regarding the use of two different implants.

\section{Differentiated shift of range of motion and postop- erative center of rotation between the groups}

The clinical and radiological parameters between the successful and unsuccessful surgical outcome groups were also compared. There were no significant differences in preoperative age and NRS value. As expected, the postoperative NRS for the successful group was significantly smaller than that of the unsuccessful group. No significant difference was found with respect to COR-X (\%) and -Y (\%) between the successful and unsuccessful groups preoperatively, whereas the unsuccessful group showed a greater degree of retained positivity for both COR-X (\%) and -Y (\%) even after MCTDR. This lesser posterior (COR-X [\%]) or caudal (COR-Y [\%]) shift of COR for the unsuccessful group was also reflected as smaller negative value for both change of COR-X (\%) and -Y (\%) even after MCTDR (Fig. 2).

The unsuccessful group showed significantly smaller C2-7 ROM and reduced angular improvement of $\mathrm{C} 2-7$ ROM postoperatively than the successful group. The unsuccessful group exhibited a more significantly reduced TDR angular improvement after surgery than did the successful group (Table 1).

\section{Correlation between center of rotation coordinates and range of motion variables}

Binary logistic regression test of radiologic variables revealed that postoperative COR-X (\%), change in COR-X (\%), postoperative COR-Y (\%), change in COR-Y (\%), and postoperative $\mathrm{C} 2-7 \mathrm{ROM}$ had statistical significance in the successful surgical outcome group (Table 2). Linear regression test shows postoperative $\mathrm{C} 2-7 \mathrm{ROM}$ was significantly related to postoperative COR-X $(\%)(p=0.038)$ (Table 3).

\section{Discussion}

\section{Importance of physiologic center of rotation restora- tion after multilevel cervical total disc replacement}

The suggested superiority of cervical TDR over conventional ACDF, which has been regarded over decades as 
Table 1. Comparison of age, NRS, and radiologic variables between successful and unsuccessful surgical outcome groups

\begin{tabular}{|c|c|c|c|}
\hline Characteristic & $\begin{array}{l}\text { Success }^{a)} \\
(\mathrm{N}=16)\end{array}$ & $\begin{array}{l}\text { Unsuccess } \\
\qquad(\mathrm{N}=8)\end{array}$ & $p$-value \\
\hline Age (yr) & $51.6 \pm 7.7$ & $53.9 \pm 9.2$ & 0.523 \\
\hline NRS at preoperation & $8.1 \pm 0.9$ & $8.0 \pm 0.8$ & 0.871 \\
\hline NRS at postoperation & $3.1 \pm 0.6$ & $5.4 \pm 0.5$ & $<0.001^{*}$ \\
\hline \multicolumn{4}{|l|}{ COR-X $(\%)^{b)}$} \\
\hline Preoperation & $34.6 \pm 20.1$ & $31.3 \pm 12.6$ & 0.679 \\
\hline Postoperation & $12.8 \pm 9.19$ & $31.7 \pm 7.21$ & $<0.001^{*}$ \\
\hline Change & $-21.7 \pm 15.9$ & $0.40 \pm 15.3$ & $0.004^{*}$ \\
\hline \multicolumn{4}{|l|}{ COR-Y $(\%)^{c)}$} \\
\hline Preoperation & $19.3 \pm 39.5$ & $13.0 \pm 42.1$ & 0.723 \\
\hline Postoperation & $-41.1 \pm 73.2$ & $31.8 \pm 70.7$ & $0.030^{*}$ \\
\hline Change & $-60.4 \pm 69.8$ & $18.8 \pm 43.3$ & $0.008^{*}$ \\
\hline \multicolumn{4}{|l|}{$\mathrm{C} 2-7 \mathrm{ROM}^{\mathrm{d}}$} \\
\hline Preoperation & $49.9 \pm 13.8$ & $49.7 \pm 13.1$ & 0.951 \\
\hline Postoperation & $51.1 \pm 7.4$ & $39.8 \pm 12.8$ & $0.027^{*}$ \\
\hline Angular change & $1.2 \pm 15.3$ & $-9.9 \pm 11.2$ & $0.037^{*}$ \\
\hline \multicolumn{4}{|l|}{ TDR ROM ${ }^{e l}$} \\
\hline Preoperation & $22.0 \pm 8.4$ & $23.8 \pm 11.2$ & 0.759 \\
\hline Postoperation & $19.3 \pm 8.2$ & $15.4 \pm 8.3$ & 0.426 \\
\hline Angular change & $-2.8 \pm 8.5$ & $-8.4 \pm 7.8$ & $0.027^{*}$ \\
\hline
\end{tabular}

Values are presented as mean \pm standard deviation.

NRS, numeric rating scale; COR, center of rotation; ROM, range of motion; TDR, total disc replacement.

${ }^{*} p<0.05$. ${ }^{\text {al }}$ Successful pain relief was defined if a $50 \%$ or more reduction of NRS score was achieved in comparison with pretreatment one. ${ }^{b}$ Percentage of the distance from COR to origin of coordinates (posterior superior corner of the lower vertebral body) along $x$-axis based on the width of the lower vertebral body. ${ }^{c}$ Percentage of the distance from COR to origin of coordinates (posterior superior corner of the lower vertebral body) along y-axis based on the height

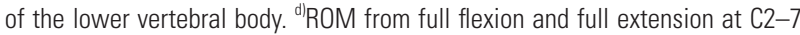
segment. ${ }^{\text {el }} \mathrm{ROM}$ from full flexion and full extension at TDR segment.

the golden standard for the surgical management of cervical radiculopathy would be based on a simple principle "promise": preservation of motion at cervical motion segment. However, those promises do not seem to be "fulfilled" for every subject who undergoes this motionpreserving cervical surgery, and increased chances for this malaccomplishment for complicated MCTDR are possible. The unexpected results after cervical TDR not only include well-manifested side effects such as implant failure or progressive immobility by heterotopic ossification but also resultant non-physiologic, exaggerated motion both at switched and adjacent levels, leading to an equivocal rate of clinical failure or adjacent segment pathology as conventional fusion surgeries. Equalizing the clinical or
Table 2. Binary logistic regression test of radiologic variables for successful surgical outcome group

\begin{tabular}{lrr} 
Variable & Value & $p$-value \\
\hline Postoperative COR-X $(\%)^{a)}$ & 12.883 & $0.005^{*}$ \\
\hline Change of COR-X $(\%)$ & 7.771 & $<0.001^{*}$ \\
\hline Postoperative COR-Y $(\%)^{b)}$ & 4.731 & $0.030^{*}$ \\
\hline Change of COR-Y $(\%)$ & 6.711 & $0.010^{*}$ \\
\hline Postoperative C2-7 ROM ${ }^{c)}$ & 6.152 & $0.013^{*}$ \\
\hline Change of C2-7 ROM & 3.114 & 0.078 \\
\hline Postoperative TDR ROM ${ }^{\mathrm{d})}$ & 1.246 & 0.264 \\
\hline Change of TDR ROM & 2.428 & 0.119 \\
\hline
\end{tabular}

COR, center of rotation; ROM, range of motion; TDR, total disc replacement. $p<0.05$. ${ }^{\text {al }}$ Percentage of the distance from COR to origin of coordinates (posterior superior corner of the lower vertebral body) along $x$-axis based on the width of the lower vertebral body. ${ }^{\text {b }}$ Percentage of the distance from COR to origin of coordinates (posterior superior corner of the lower vertebral body) along y-axis based on the height of the lower vertebral body. ${ }^{\text {cl}} \mathrm{ROM}$ from full flexion and full extension at C2-7 segment. ${ }^{\text {dl }}$ ROM from full flexion and full extension at TDR segment.

radiological consequences after cervical TDR with those of ACDF has brought up emphasis on the issue of preservation of "quality" of motion at original cervical motion segment, including maintenance of physiological COR, rather than the mere provision of adequate magnitude or "quantity" of ROM after cervical TDR.

\section{Initial center of rotation in comparison to previous references}

This study presented that mean pre-MCTDR COR-X and -Y were $33.5 \% \pm 17.7 \%$ and $17.2 \% \pm 39.6 \%$, respectively. This suggested that pre-MCTDR COR was placed about one third posterior of and higher than superior endplate of the lower cervical vertebrae. Another study investigating COR placement with the same measurement method after single-level TDR showed that the mean preoperative COR-X and $-\mathrm{Y}$ were $41.85 \% \pm 15.75 \%$ and $-12.59 \% \pm 26.35 \%$, respectively, indicating that mean COR was located at slightly inferior and posterior to the middle of the superior endplate of the lower vertebral body [10] This discrepancy is due to the preoperative COR being widely dispersed along the various coordinates and is vulnerable for variation according to the degree of pathologic process within cervical motion segment beyond normal ranges [14]. It was reported that the mean COR had shifted more anteriorly and cranially as the person aged even in asymptomatic subjects. A significant difference was 
Table 3. Linear regression analysis between COR-X $(\%)^{a)}$, $-Y(\%)^{b}$ and postoperative C2-7 ROM ${ }^{c l}$ and TDR ROM ${ }^{d l}$

\begin{tabular}{|c|c|c|c|c|c|}
\hline \multirow{2}{*}{ Variable } & \multicolumn{2}{|c|}{ Unstandadized coefficient } & \multirow{2}{*}{ Standadized coefficient $(\beta)$} & \multirow{2}{*}{$t$-value } & \multirow{2}{*}{$p$-value } \\
\hline & $B$ & Standard error & & & \\
\hline \multicolumn{6}{|l|}{ Postoperative C2-7 ROM } \\
\hline Postoperative COR-X (\%) & -45.064 & 20.162 & -0.518 & -2.235 & $0.038^{*}$ \\
\hline Postoperative COR-Y (\%) & 5.645 & 5.665 & 0.415 & 0.996 & 0.332 \\
\hline Change of COR-X $(\%)$ & 10.788 & 13.451 & 0.188 & 0.802 & 0.432 \\
\hline Change of COR-Y (\%) & -6.607 & 6.273 & -0.443 & -1.053 & 0.305 \\
\hline \multicolumn{6}{|l|}{ Postoperative TDR ROM } \\
\hline Postoperative COR-X (\%) & -5.637 & 16.265 & -0.085 & -0.347 & 0.733 \\
\hline Postoperative COR-Y (\%) & -6.532 & 4.570 & -0.626 & -1.429 & 0.169 \\
\hline Change of COR-X $(\%)$ & -9.136 & 10.851 & -0.207 & -0.842 & 0.410 \\
\hline Change of COR-Y (\%) & 6.433 & 5.060 & 0.562 & 1.271 & 0.219 \\
\hline
\end{tabular}

COR, center of rotation; ROM, range of motion; TDR, total disc replacement.

$" p<0.05{ }^{\text {al }}$ Percentage of the distance from COR to origin of coordinates (posterior superior corner of the lower vertebral body) along $x$-axis based on the width of the lower vertebral body. ${ }^{\text {be }}$ ercentage of the distance from COR to origin of coordinates (posterior superior corner of the lower vertebral body) along y-axis based on the

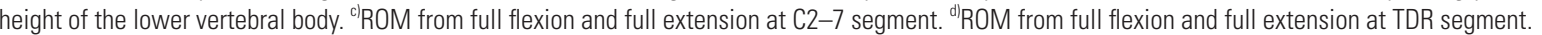

found in COR-Y of patients $>50$ years old compared with those of patients $<50$ years old [13]. Our study included candidates for MCTDR whose mean age was about 52 years with multilevel disc degeneration. Multiple involved cervical levels may present a more advanced degenerative process and further deviated COR than previously reported single-level disc degeneration series, suggesting more anterior and cranial positions for COR in our series compared with those reported in other studies [13]. Thus, more posterior and caudal shift of COR by MCTDR was closely related to successful surgical outcome by transferring $\mathrm{COR}$ closer to the normal position in theory for our series.

\section{The relationship between center of rotation restora- tion and clinical results}

Although reference on the location of COR comparison between successful and unsuccessful clinical results after cervical MCTDR is not available, previous literature has shown the influence of COR on sagittal ROM after singlelevel TDR. Rong et al. [10] have reported that less change of COR-X after TDR correlated well with preservation of cervical ROM after TDR. This result indicated that minimized change or preservation of original COR was crucial for the maintenance of cervical ROM after TDR [10]. Another study on lumbosacral TDR regarding relationship between COR change and clinical outcomes also demon- strated that the difference between patients' inherent COR and post-TDR COR was associated with better clinical outcomes. Maintaining original individual preoperative COR even after lumbosacral TDR switch helped to accomplish good clinical outcomes [15].

\section{Interpretation for discrepant center of rotation shift from previous references}

Previous studies have presented discrepant ventrocranial shifts of COR after single-level cervical TDR, contradictory to the results from our study $[8,10]$. Several hypotheses (or assumptions) could be applied for the interpretation of this contradicting phenomenon.

(1) The authors assumed that the pre-TDR CORs of those literatures were not deviated so much as compared with our study, because those studies are based on the surgical indication of TDR for candidates mostly suffering from single-level degenerative disc disease. Unfortunately, our series dealing with MCTDR would be more vulnerable to an inherently more dispersed COR coordinates since we have dealt with progressed degenerative disc diseases affecting multiple segments.

(2) This discrepant COR adjustment to a rather "dorsocaudal" location in proximity to normal position in $\mathrm{x}$ and $y$-axis in our series to previous references could also be attributed to the difference of prosthesis design. While the postoperative COR tend to shift to cranial direction 
after the insertions of prostheses composed with the ball part of the core implanted on the upper plates of devices, it tended to shift in counter, caudal direction with the prostheses composed of the ball implanted on the lower plates (e.g., Prodisc-C Vivo or Baguera C included in this series) $[16,17]$. Therefore, the need for COR adjustment to a "dorsocaudal" location in proximity to normal coordinates as in our series has mandated the authors to use the latter type of the prosthesis.

(3) Forward shift of COR after cervical TDR might also be released after insufficient depth of prosthesis implantation [8]. Ventrally located core of the prosthesis from the operating surgeon's fear of possibly impinging the ventral thecal sac during device insertion could have played a role for this forward shift of postoperative COR in the prior studies.

Our series has manifested that both achievement of more caudal and posterior COR shift and preservation of postoperative ROM subsequently yield better clinical results after MCTDR. In this regard, increased postoperative ROM was closely related to posterior shift of postoperative COR-X (\%). One clinical study showed similar results; larger anterior displacement of COR after TDR was related to decreased ROM of TDR level [8]. The study using finite element analysis demonstrated that the backward shift of COR-X could increase ROM of corresponding segments, whereas the forward shift would decrease ROM [18]. It was reported that the insufficient depth of prosthesis implantation and consequent forward shift of COR produced limited ROM of cervical and TDR segment $[19,20]$. In conclusion, posterior placement of COR was an important factor to preserve postoperative ROM and to further accomplish successful clinical outcome.

This study had several limitations. First, this investigation was performed in a retrospective manner with a limited number of patients ( $n=24)$, weakening the statistical findings of this study. Second, the sample size of this series was small, and the mean follow-up period was relatively short ( $<2$ years). Authors are currently continuing this series to elongate the follow-up period and recruit additional candidates to reach the ideal standards for the assessment of MCTDR operative results.

\section{Conclusions}

The preoperative CORs of the patients with multiple cervical disc degeneration were located at relative ventral and cranial coordinates inside cervical motion segment compared with normal physiological range. Therefore, a dorsal, caudal iatrogenic shift of COR in close proximity to the original coordinate would be crucial for a successful clinical outcome achievement after MCTDR. Successful outcome subjects showed angular preservation of whole C2-7 and MCTDR segment compared with unsuccessful outcome subjects. The position of COR at $\mathrm{x}$-axis after MCTDR was a key factor in determining C2-7 ROM maintenance.

\section{Conflict of Interest}

No potential conflict of interest relevant to this article was reported.

\section{ORCID}

Jung Hwan Lee: https://orcid.org/0000-0003-2680-6953

Jun Ho Lee: https://orcid.org/0000-0001-6246-8053

\section{References}

1. Wu AM, Xu H, Mullinix KP, et al. Minimum 4-year outcomes of cervical total disc arthroplasty versus fusion: a meta-analysis based on prospective randomized controlled trials. Medicine (Baltimore) 2015;94:e665.

2. Daentzer D, Welke B, Hurschler C, et al. In vitroanalysis of kinematics and intradiscal pressures in cervical arthroplasty versus fusion: a biomechanical study in a sheep model with two semi-constrained prosthesis. Biomed Eng Online 2015;14:27.

3. Peng CW, Yue WM, Basit A, et al. Intermediate results of the Prestige LP cervical disc replacement: clinical and radiological analysis with minimum twoyear follow-up. Spine (Phila Pa 1976) 2011;36:E10511.

4. Pandey PK, Pawar I, Gupta J, Verma RR. Comparison of outcomes of single-level anterior cervical discectomy with fusion and single-level artificial cervical disc replacement for single-level cervical degenerative disc disease. Spine (Phila Pa 1976) 2017;42:E419.

5. Chen F, Yang J, Ni B, Guo Q, Lu X, Xie N. Clinical and radiological follow-up of single-level Prestige LP cervical disc replacement. Arch Orthop Trauma Surg 
2013;133:473-80.

6. Matsunaga S, Kabayama S, Yamamoto T, Yone K, Sakou T, Nakanishi K. Strain on intervertebral discs after anterior cervical decompression and fusion. Spine (Phila Pa 1976) 1999;24:670-5.

7. Lopez-Espina CG, Amirouche F, Havalad V. Multilevel cervical fusion and its effect on disc degeneration and osteophyte formation. Spine (Phila Pa 1976) 2006;31:972-8.

8. Lou J, Li H, Rong X, Wu W, Liu H. Location change of center of rotation after single-level cervical total disc replacement with ProDisc-C. Acta Orthop Traumatol Turc 2016;50:339-45.

9. Mo Z, Zhao Y, Du C, Sun Y, Zhang M, Fan Y. Does location of rotation center in artificial disc affect cervical biomechanics? Spine (Phila Pa 1976) 2015;40:E469-75.

10. Rong X, Gong Q, Liu H, et al. The effect of deviated center of rotation on flexion-extension range of motion after single-level cervical arthroplasty: an in vivo study. Spine (Phila Pa 1976) 2014;39(26 Spec No.):B12-8.

11. Kim SW, Paik SH, Oh JK, Kwak YH, Lee HW, You $\mathrm{KH}$. The impact of coronal alignment of device on radiographic degeneration in the case of total disc replacement. Spine J 2016;16:470-9.

12. Manchikanti L, Singh V, Cash KA, Pampati V, Datta S. A comparative effectiveness evaluation of percutaneous adhesiolysis and epidural steroid injections in managing lumbar post surgery syndrome: a randomized, equivalence controlled trial. Pain Physician 2009;12:E355-68.

13. Liu B, Liu Z, VanHoof T, Kalala J, Zeng Z, Lin X. Kinematic study of the relation between the instan- taneous center of rotation and degenerative changes in the cervical intervertebral disc. Eur Spine J 2014;23:2307-13.

14. Amevo B, Aprill C, Bogduk N. Abnormal instantaneous axes of rotation in patients with neck pain. Spine (Phila Pa 1976) 1992;17:748-56.

15. Lee CS, Lee DH, Hwang CJ, Kim H, Noh H. The effect of a mismatched center of rotation on the clinical outcomes and flexion-extension range of motion: lumbar total disk replacement using mobidisc at a 5.5-year follow-up. J Spinal Disord Tech 2014;27:14853.

16. Rousseau MA, Bonnet X, Skalli W. Influence of the geometry of a ball-and-socket intervertebral prosthesis at the cervical spine: a finite element study. Spine (Phila Pa 1976) 2008;33:E10-4.

17. Kowalczyk I, Lazaro BC, Fink M, Rabin D, Duggal N. Analysis of in vivo kinematics of 3 different cervical devices: Bryan disc, ProDisc-C, and Prestige LP disc. J Neurosurg Spine 2011;15:630-5.

18. Galbusera F, Anasetti F, Bellini CM, Costa F, Fornari $M$. The influence of the axial, antero-posterior and lateral positions of the center of rotation of a ball-and-socket disc prosthesis on the cervical spine biomechanics. Clin Biomech (Bristol, Avon) 2010;25:397-401.

19. Colle KO, Butler JB, Reyes PM, Newcomb AG, Theodore N, Crawford NR. Biomechanical evaluation of a metal-on-metal cervical intervertebral disc prosthesis. Spine J 2013;13:1640-9.

20. Kelly BP, Zufelt NA, Sander EJ, DiAngelo DJ. The influence of fixed sagittal plane centers of rotation on motion segment mechanics and range of motion in the cervical spine. J Biomech 2013;46:1369-75. 